\title{
Basic Aspects of Geopotential Field Approximation From Satellite-to-Satellite Tracking Data
}

\author{
W. Freeden, V. Michel \\ University of Kaiserslautern \\ Laboratory of Technomathematics \\ Geomathematics Group \\ P.O.Box 3049 \\ 67653 Kaiserslautern \\ Germany \\ email: freeden@mathematik.uni-kl.de \\ michel@mathematik.uni-kl.de \\ www:http://www.mathematik.uni-kl.de/ wwwgeo/...
}

\begin{abstract}
The satellite-to-satellite tracking (SST) problems are characterized from mathematical point of view. Uniqueness results are formulated. Moreover, the basic relations are developed between (scalar) approximation of the earth's gravitational potential by 'scalar basis systems' and (vectorial) approximation of the gravitational field by 'vectorial basis systems'. Finally, the mathematical justification is given for approximating the external geopotential field by finite linear combinations of certain gradient fields (for example, gradient fields of multi-poles) consistent to a given set of SST data.
\end{abstract}

AMS classification: Primary: 86A20, 86A30. Secondary: 31B05, 35J05.

Key Words: satellite-to-satellite tracking, external gravitational field, uniqueness, fundamental systems, scalar and vectorial trial systems, closure theorems. 


\section{The SST Problems}

The purpose of high-low satellite-to-satellite tracking (hi-lo SST) by use of the Global Positioning System (GPS) (as realized e.g. by the recently launched German satellite CHAMP (2000) of the GeoForschungsZentrum (GFZ) Potsdam) is to develop the geopotential field from measured ranges (geometrical distances) between a low earth orbiter (LEO) and the high-flying GPSsatellites. In this paper, hi-lo SST is discussed from mathematical point of view as the problem of determining the external gravitational field of the earth from a given set of gradient vectors at the altitude of the low earth orbiter (LEO).

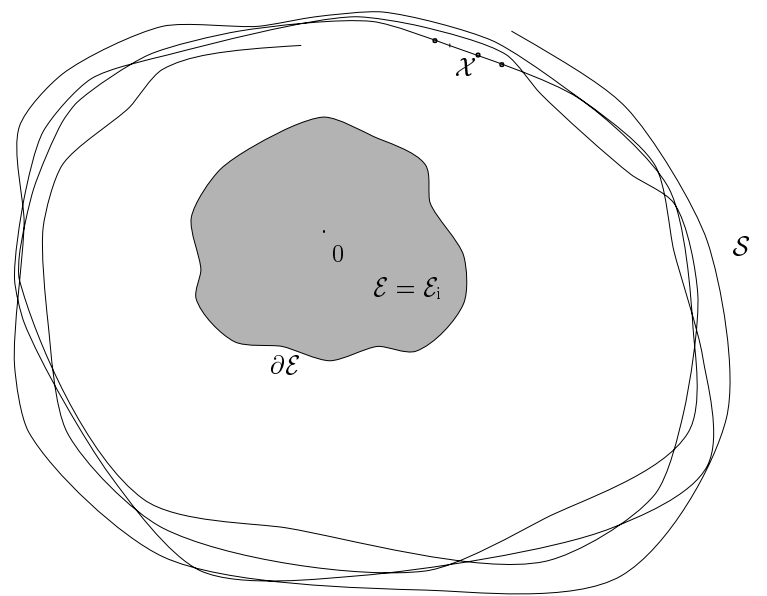

Figure 1: Illustration of the sets $\mathcal{E}$ and $\mathcal{S}$

In order to translate hi-lo SST into a mathematical formulation (see [7], for alternative approaches $[3,4,9,17,18,19,22])$ we start from the following geometrical situation (cf. Figure 1): Let the surface $\partial \mathcal{E}$ of the earth $\mathcal{E}$ and the orbital set $\mathcal{S}$ of the low earth orbiter (LEO) be given in such a way that $\mathcal{S}$ is a strict subset of the earth's exterior $\mathcal{E}_{\mathrm{e}}$ satisfying

$$
\sup _{x \in \partial \mathcal{E}}|x|<h=\inf _{x \in \mathcal{S}}|x| \text {. }
$$

The arrangement of the GPS-satellites is such that at least four satellites are simultaneously visible above the horizon anywhere on the earth's surface $\partial \mathcal{E}$ and the orbit $\mathcal{S}$ of the low earth orbiter as well, all the time. Moreover, the GPS-satellites are supposed to be placed in six circular orbits $\Omega_{\gamma_{i}}$ of radii $\gamma_{i}, i=1, \ldots, 6$, around the origin with $\gamma_{i} \gg h, i=1, \ldots, 6$; and $n$ be the total 
number of GPS-satellites. To every LEO-position $x \in \mathcal{S}$, therefore, there exist at least $m(\geq 4)$ visible GPS-satellites located at $y_{l_{1}}, \ldots, y_{l_{m}}, l_{i} \in\{1, \ldots, n\}$ for $i=1, \ldots, m$, such that the geometrical distances (ranges) $d_{l_{i}}=\left|x-y_{l_{i}}\right|$, $l_{i} \in\{1, \ldots, n\}$ for $i=1, \ldots, m$, are measurable. Since the orbits of the GPSsatellites are assumed to be known, the coordinates of the low earth orbiter (LEO) located at $x \in \mathcal{S}$ can be derived from simultaneous range measurements to the satellites. From this the relative positions of the satellites at $x$ and $y_{l_{i}}$, i.e. $p_{l_{i}}=x-y_{l_{i}}, l_{i} \in\{1, \ldots, n\}$ for $i=1, \ldots, m$, become available at time $t$. The relative velocities $v_{l_{i}}$ and accelerations $a_{l_{i}}$ are obtainable by differentiating the relative positions with respect to $t$. We may assume that the measurements are produced at a sufficiently dense rate so that (numerical) differentiation can be performed without any difficulty. The interesting expressions now are the relative accelerations $a_{l_{i}}, i=1, \ldots, m$, all of which are determined for inertial motion (in accordance with the Newton-Euler equation) by the gravitational field only and may be equated by the difference of the gradient field of the geopotential, $V$, here evaluated at the locations of $x$ and $y_{l_{i}}, l_{i} \in\{1, \ldots, n\}$ for $i=1, \ldots, m$. To be more specific,

$$
a_{l_{i}}(x)=(\nabla V)(x)-(\nabla V)\left(y_{l_{i}}\right), \quad x \in \mathcal{S},
$$

$i=1, \ldots, m$. (Note that the gravitational force is considered now to be independent of time $t$ at a certain position. In other words, we assume here that the time-like variations of the field are so slow as to be negligible.) From (2) it follows that

$$
(\nabla V)(x)=\sum_{i=1}^{m} \alpha_{i}\left(a_{l_{i}}(x)+(\nabla V)\left(y_{l_{i}}\right)\right), \quad x \in \mathcal{S}
$$

for all selections $\left(\alpha_{1}, \ldots, \alpha_{m}\right)^{T} \in \mathbb{R}^{m}$ satisfying $\sum_{i=1}^{m} \alpha_{i}=1$. The influence of the Global Positioning System (GPS) to the choice of the coefficients $\alpha_{1}, \ldots, \alpha_{m}$ will not be investigated here. (Usually, in practice, $(\nabla V)\left(y_{l_{i}}\right)$ are supposed to be so small as to be negligible).

Loosely spoken, the mathematical formulation of the hi-lo SST problem now reads as follows:

Let there be known the gradient vectors $v(x)=(\nabla V)(x), x \in \mathcal{X}$, for a subset $\mathcal{X} \subset \mathcal{S}$ of points at the flight positions of the low earth orbiter (LEO). Find an approximation $u$ of the geopotential field $v$ on $\overline{\mathcal{E}_{\mathrm{e}}}=\partial \mathcal{E} \cup \mathcal{E}_{\mathrm{e}}$, i.e. on and outside the earth's surface, such that the geopotential field $v$ and its approximation $u$ are in $\varepsilon$-accuracy on $\overline{\mathcal{E}_{\mathrm{e}}}$ (with respect to the uniform topology in $\overline{\mathcal{E}_{\mathrm{e}}}$ ) so that $v(x)=u(x)$ for all $x \in \mathcal{X}$. 
As a matter of fact, the approximation property will be proved in this paper even with respect to the Hölder-topology in $\overline{\mathcal{E}_{\mathrm{e}}}$.

The problem of knowing the vectors $(\nabla V)\left(y_{i}\right), i=1, \ldots, m$, in (2) is not relevant anymore, if low-low satellite-to-satellite tracking (briefly, lo-lo SST) will be used (as planned by the future GFZ-NASA 'two satellite configuration' GRACE (2002)). By the tandem mode procedure of lo-lo SST (cf. [3, 4]) the vectors $a_{l_{i}}, i=1, \ldots, m$, are measurable at two different positions $x$ and $x^{*}$ with $x^{*}=x+h(x), x \in \mathcal{S}$, where $h: \mathcal{S} \rightarrow \mathbb{R}^{3}$ is the difference vector field between the two satellite positions (i.e. $|h(x)| \geq \iota>0$ with $\iota$ denoting the intersatellite range). Consequently, the mathematical scenario of the lo-lo SST problem is characterized as follows:

Let there be known the vectors $v(x)=(\nabla V)(x)$ and $\tilde{v}(x)=v(x+h(x))=$ $(\nabla V)(x+h(x)), x \in \mathcal{X}$, for a subset $\mathcal{X} \subset \mathcal{S}$. Find an approximation $u$ of $v$ on $\overline{\mathcal{E}_{\mathrm{e}}}$, such that $v$ and $u$ are in $\varepsilon$-accuracy (with respect to the uniform topology in $\left.\overline{\mathcal{E}_{\mathrm{e}}}\right)$, so that $v(x)-v(x+h(x))=u(x)-u(x+h(x))$ for all $x \in \mathcal{X}$.

\section{Notational Background}

Let us begin by introducing some notations that will be used throughout this paper.

We consider $\mathbb{R}^{3}$ to be equipped with the canonical inner product $\cdot$ and the associated norm || . Using $\varepsilon^{1}, \varepsilon^{2}, \varepsilon^{3}$ as canonical orthonormal basis in $\mathbb{R}^{3}$ each element $x \in \mathbb{R}^{3}$ may be represented in cartesian coordinates as follows: $x=\Sigma_{i=1}^{3}\left(x \cdot \varepsilon^{i}\right) \varepsilon^{i}$.

If $\mathcal{G}$ is a set of points in $\mathbb{R}^{3}, \partial \mathcal{G}$ will denote its boundary. The set $\mathcal{G}=\mathcal{G} \cup \partial \mathcal{G}$ will be called the closure of $\mathcal{G}$. A set $\mathcal{G} \subset \mathbb{R}^{3}$ will be called a region if it is open and connected.

The restriction of a function $f$ to a subset $M$ of its domain is denoted by $f \mid M$, for a set $L$ of functions we set $L \mid M=\{f|M| f \in L\}$.

A function $f$ possessing $k$ ( $\lambda$-Hölder) continuous derivatives is said to be of class $C^{(k)}\left(C^{(k, \lambda)}\right)$ (note that we use $C^{(k)}, C^{(k, \lambda)}$ simultaneously for scalar- and vector-valued functions).

A region $\mathcal{G}$ will be called regular, if its boundary $\partial \mathcal{G}$ satisfies the following properties:

(i) $\partial \mathcal{G}$ divides the three-dimensional Euclidean space $\mathbb{R}^{3}$ into the bounded region $\mathcal{G}=\mathcal{G}_{\mathrm{i}}$ (inner space) and the unbounded region $\mathcal{G}_{\mathrm{e}}$ (outer space) defined by $\mathcal{G}_{\mathrm{e}}=\mathbb{R}^{3} \backslash \overline{\mathcal{G}}$. 
(ii) $\partial \mathcal{G}$ is a closed and compact surface with no double points.

(iii) The origin 0 is contained in $\mathcal{G}_{\mathrm{i}}$.

(vi) $\partial \mathcal{G}$ is a $C^{(2, \lambda)}$-surface, i.e. $\partial \mathcal{G}$ is locally $C^{(2, \lambda)}$-smooth.

From this definition it is clear that all (geophysically relevant) earth's models are included. Regular regions are, for example, the inner space of a sphere, an ellipsoid, a geoid, and a (sufficiently smooth) real earth's surface.

$\operatorname{Pot}\left(\mathcal{G}_{\mathrm{e}}\right)$ denotes the space of functions $V: \mathcal{G}_{\mathrm{e}} \rightarrow \mathbb{R}$ with the following properties:

(i) $V$ is twice continuously differentiable in $\mathcal{G}_{\mathrm{e}}: V \in C^{(2)}\left(\mathcal{G}_{\mathrm{e}}\right)$,

(ii) $V$ satisfies Laplace's equation in $\mathcal{G}_{\mathrm{e}}: \Delta V=0$ in $\mathcal{G}_{\mathrm{e}}$,

(iii) $V$ is regular at infinity:

$$
|V(x)|=O\left(\frac{1}{|x|}\right), \quad|\nabla V(x)|=O\left(\frac{1}{|x|^{2}}\right), \quad|x| \rightarrow \infty .
$$

We denote by $\operatorname{Pot}^{(k)}\left(\overline{\mathcal{G}_{\mathrm{e}}}\right)$ the space of all functions $V: \overline{\mathcal{G}_{\mathrm{e}}} \rightarrow \mathbb{R}$ such that $V$ is a member of class $C^{(k)}\left(\overline{\mathcal{G}_{\mathrm{e}}}\right)$ and $V \mid \mathcal{G}_{\mathrm{e}}$ satisfies, in addition, the properties (i), (ii), (iii) of a function of class $\operatorname{Pot}\left(\mathcal{G}_{\mathrm{e}}\right)$. Briefly formulated,

$$
\operatorname{Pot}^{(k)}\left(\overline{\mathcal{G}_{\mathrm{e}}}\right)=\operatorname{Pot}\left(\mathcal{G}_{\mathrm{e}}\right) \cap C^{(k)}\left(\overline{\mathcal{G}_{\mathrm{e}}}\right) .
$$

Furthermore, we set

$$
\operatorname{Pot}^{(k, \lambda)}\left(\overline{\mathcal{G}_{\mathrm{e}}}\right)=\operatorname{Pot}\left(\mathcal{G}_{\mathrm{e}}\right) \cap C^{(k, \lambda)}\left(\overline{\mathcal{G}_{\mathrm{e}}}\right) .
$$

By $\operatorname{pot}\left(\mathcal{G}_{\mathrm{e}}\right)$ we denote the space of vector fields $v: \mathcal{G}_{\mathrm{e}} \rightarrow \mathbb{R}^{3}$ satisfying the following properties:

(i) $v$ is continuously differentiable in $\mathcal{G}_{\mathrm{e}}: v \in C^{(1)}\left(\mathcal{G}_{\mathrm{e}}\right)$,

(ii) $v$ is a harmonic vector field in $\mathcal{G}_{\mathrm{e}}$ :

$$
\operatorname{div} v=0, \quad \operatorname{curl} v=0 \quad \text { in } \mathcal{G}_{\mathrm{e}},
$$

(iii) $v$ is regular at infinity:

$$
|v(x)|=o(1) \quad|x| \rightarrow \infty .
$$


In analogy to the scalar approach we let

$$
\operatorname{pot}^{(k)}\left(\overline{\mathcal{G}_{\mathrm{e}}}\right)=\operatorname{pot}\left(\mathcal{G}_{\mathrm{e}}\right) \cap C^{(k)}\left(\overline{\mathcal{G}_{\mathrm{e}}}\right)
$$

and

$$
\operatorname{pot}^{(k, \lambda)}\left(\overline{\mathcal{G}_{\mathrm{e}}}\right)=\operatorname{pot}\left(\mathcal{G}_{\mathrm{e}}\right) \cap C^{(k, \lambda)}\left(\overline{\mathcal{G}_{\mathrm{e}}}\right) .
$$

As is well-known, every $v \in \operatorname{pot}\left(\mathcal{G}_{\mathrm{e}}\right)$ can be represented as gradient field $v=$ $\nabla V$, where $V$ is of class $\operatorname{Pot}\left(\mathcal{G}_{\mathrm{e}}\right.$ ), and vice versa (see, for example, [14]).

$C^{(0, \lambda)}\left(\partial \mathcal{G}_{\mathrm{e}}\right)$ is a (non-complete) normed space with the norm defined by

$$
\|F\|_{0}\left(\partial \mathcal{G}_{\mathrm{e}}\right)=\sup _{x \in \partial \mathcal{G}_{\mathrm{e}}}|F(x)|
$$

and a Banach space with

$$
\|F\|_{\lambda}\left(\partial \mathcal{G}_{\mathrm{e}}\right)=\sup _{x \in \partial \mathcal{G}_{\mathrm{e}}}|F(x)|+\sup _{\substack{x, y \in \partial \mathcal{G}_{\mathrm{e}} \\ x \neq y}} \frac{|F(x)-F(y)|}{|x-y|^{\lambda}}, 0<\lambda<1 .
$$

$C^{(0, \lambda)}\left(\partial \mathcal{G}_{\mathrm{e}}\right)$ is a (non-complete) normed space with \|\|$_{\mu}$ for $\mu<\lambda$ (see e.g. $[20])$.

In $C^{(0, \lambda)}\left(\partial \mathcal{G}_{\mathrm{e}}\right)$ we have the $\left(L^{2}-\right)$ inner product

$$
<F, G>_{\partial \mathcal{G}_{\mathrm{e}}}=\int_{\partial \mathcal{G}_{\mathrm{e}}} F(x) G(x) d S(x),
$$

where $d S(x)$ (or, when confusion is not likely to arise, $d S$ ) denotes the surface element. The inner product $<,>_{\partial \mathcal{G}_{\mathrm{e}}}$ implies the norm

$$
|F|\left(\partial \mathcal{G}_{\mathrm{e}}\right)=\sqrt{<F, F>\partial_{\mathcal{e}}} .
$$

The space $\left(C^{(0, \lambda)}\left(\partial \mathcal{G}_{\mathrm{e}}\right),<,>_{\partial \mathcal{G}_{\mathrm{e}}}\right)$ is a pre-Hilbert space. For every function $F \in C^{(0, \lambda)}\left(\partial \mathcal{G}_{\mathrm{e}}\right)$ we have the norm-estimate

$$
|F|\left(\partial \mathcal{G}_{\mathrm{e}}\right) \leq C\|F\|_{0}\left(\partial \mathcal{G}_{\mathrm{e}}\right) \leq C\|F\|_{\lambda}\left(\partial \mathcal{G}_{\mathrm{e}}\right)
$$

where

$$
C=\left(\int_{\partial \mathcal{G}_{\mathrm{e}}} d S\right)^{1 / 2}
$$

By $L^{2}\left(\partial \mathcal{G}_{\mathrm{e}}\right)$ we denote the space of (Lebesgue) square-integrable functions on the boundary $\partial \mathcal{G}_{\mathrm{e}} . L^{2}\left(\partial \mathcal{G}_{\mathrm{e}}\right)$ is a Hilbert space with respect to the inner product $<,>_{\partial \mathcal{G}_{\mathrm{e}}}$ and a Banach space with respect to the norm ||$\left(\partial \mathcal{G}_{\mathrm{e}}\right), L^{2}\left(\partial \mathcal{G}_{\mathrm{e}}\right)$ is the completion of $C^{(0)}\left(\partial \mathcal{G}_{\mathrm{e}}\right)$ (and of $\left.C^{(0, \lambda)}\left(\partial \mathcal{G}_{\mathrm{e}}\right)\right)$ with respect to the norm ||$\left(\partial \mathcal{G}_{\mathrm{e}}\right)$.

For later use we introduce the concept of fundamental systems: 
Definition 2.1. A system $\mathcal{Y}=\left(y_{n}\right)_{n=0,1, \ldots} \subset \mathcal{G}_{\mathrm{i}}$ is called a fundamental system in $\mathcal{G}_{\mathrm{i}}$, if $F: \mathcal{G}_{\mathrm{i}} \rightarrow \mathbb{R}$ with $F \in C^{(2)}\left(\mathcal{G}_{\mathrm{i}}\right), \Delta F=0$ in $\mathcal{G}_{\mathrm{i}}$, and $F\left(y_{n}\right)=0$ for $n=0,1, \ldots$ implies $F=0$ in $\mathcal{G}_{\mathrm{i}}$. Analogously, a system $\mathcal{Y}=\left(y_{n}\right)_{n=0,1, \ldots} \subset \mathcal{G}_{\mathrm{e}}$ is called a fundamental system in $\mathcal{G}_{\mathrm{e}}$, if $F: \mathcal{G}_{\mathrm{e}} \rightarrow \mathbb{R}$ with $F \in C^{(2)}\left(\mathcal{G}_{\mathrm{e}}\right), \Delta F=0$ in $\mathcal{G}_{\mathrm{e}}, F$ is regular at infinity, and $F\left(y_{n}\right)=0$ for $n=0,1, \ldots$ implies $F=0$ in $\mathcal{G}_{\mathrm{e}}$.

\section{Uniqueness of the SST Problems}

Our considerations start with the problem of uniqueness corresponding to an infinite system $\mathcal{X} \subset \mathcal{S}$ of known GPS-SST data.

THEOREM 3.1. Let $\mathcal{E}=\mathcal{E}_{\mathrm{i}}$ (i.e. the inner space of the earth) be a regular region. Suppose that $\mathcal{X}$ (i.e. the subset of observational points of the satellite orbit $\mathcal{S})$ is a fundamental system in $\mathcal{E}_{\mathrm{e}}$. If $v$ is of class $\operatorname{pot}^{(0)}\left(\overline{\mathcal{E}_{\mathrm{e}}}\right)$ with

$$
v(x)=0, \quad x \in \mathcal{X}
$$

then $v=0$ in $\overline{\mathcal{E}_{\mathrm{e}}}$.

Proof. Any field $v \in \operatorname{pot}\left(\mathcal{E}_{\mathrm{e}}\right)$ can be expressed in the form $\nabla V$, hence, the coordinate functions $v \cdot \varepsilon^{i}, i=1,2,3$, satisfy $\Delta\left(v \cdot \varepsilon^{i}\right)=\Delta\left(\left(\varepsilon^{i} \cdot \nabla\right) V\right)=$ $\left(\varepsilon^{i} \cdot \nabla\right) \Delta V=0$ in $\mathcal{E}_{\mathrm{e}}$. Moreover, according to our assumption, $\left(\varepsilon^{i} \cdot \nabla\right) V(x)=0$ for all points $x$ of the fundamental system $\mathcal{X}$ in $\mathcal{E}_{\mathrm{e}}$. This implies $v \cdot \varepsilon^{i}=0$ in $\mathcal{E}_{\mathrm{e}}, i=1,2,3$, as required.

In other words, the earth's external gravitational field is uniquely detectable on and outside the earth's surface $\partial \mathcal{E}$ from GPS-SST data corresponding to a system of gradient vectors determined on a fundamental system $\mathcal{X}$ on the satellite orbit $\mathcal{S}$.

Furthermore we are able to verify the following result (for a similar theorem see [7]).

THEOREM 3.2. Let $\mathcal{E}_{\mathrm{i}}$ be a regular region. Suppose that $\mathcal{X}$ is a fundamental system in $\mathcal{E}_{\mathrm{e}}$ with $\sup _{x \in \partial \mathcal{E}_{\mathrm{e}}}|x|<h \leq \inf _{x \in \mathcal{X}}|x|$. If $v$ is a field of class $\operatorname{pot}^{(0)}\left(\overline{\mathcal{E}_{\mathrm{e}}}\right)$ with

$$
-\frac{x}{|x|} \cdot v(x)=0, \quad x \in \mathcal{X},
$$

then $v=0$ in $\overline{\mathcal{E}_{\mathrm{e}}}$. 
Proof. Again we base our arguments on the identity $v=\nabla V$ in $\mathcal{E}_{\mathrm{e}}$. From our assumptions it is clear that there exists a sphere $\partial \mathcal{A}$ with radius $\alpha$ around the origin such that $\sup _{x \in \partial \mathcal{E}}|x|<\alpha<h$, i.e. $\mathcal{A}_{\mathrm{e}}$ is a strict subset of $\mathcal{E}_{\mathrm{e}}$. Outside the sphere $\partial \mathcal{A}$ the potential $V \in \operatorname{Pot}\left(\mathcal{E}_{\mathrm{e}}\right)$ may be expanded in terms of outer harmonics (see e.g. $[14,15])$ :

$$
V(x)=\sum_{n=0}^{\infty} \sum_{k=1}^{2 n+1} V^{\wedge}(n, k) H_{n, k}(\alpha ; x), \quad x \in \mathcal{A}_{\mathrm{e}},
$$

where

$$
H_{n, k}(\alpha ; x)=\frac{1}{\alpha}\left(\frac{\alpha}{|x|}\right)^{n+1} Y_{n, k}\left(\frac{x}{|x|}\right),
$$

$\left(Y_{n, k}\right)_{\substack{n=0,1, \ldots, k=1, \ldots, 2 n+1}}$ is an $\mathcal{L}^{2}$-orthonormal system of spherical harmonics on the unit sphere, and $V^{\wedge}(n, k)$ are the orthogonal coefficients

$$
V^{\wedge}(n, k)=\int_{\partial \mathcal{A}_{\mathrm{e}}} V(x) H_{n, k}(\alpha ; x) d S,
$$

$n=0,1, \ldots ; k=1, \ldots, 2 n+1$. It is not difficult to see that

$$
-\frac{x}{|x|} \cdot(\nabla V)(x)=\sum_{n=0}^{\infty} \sum_{k=1}^{2 n+1} \frac{n+1}{|x|} V^{\wedge}(n, k) H_{n, k}(\alpha ; x), \quad x \in \mathcal{A}_{\mathrm{e}} .
$$

In accordance with our assumption $-\frac{x}{|x|} \cdot(\nabla V)(x)=0, x \in \mathcal{X}$, we thus obtain

$$
\sum_{n=0}^{\infty} \sum_{k=1}^{2 n+1} V^{\wedge}(n, k)(n+1) H_{n, k}(\alpha ; x)=0, \quad x \in \mathcal{X} .
$$

Since $\mathcal{X}$ is a fundamental system in $\mathcal{A}_{\mathrm{e}},(5)$ holds true in $\mathcal{A}_{\mathrm{e}}$. The theory of spherical harmonics then tells us that $V^{\wedge}(n, k)(n+1)=0$, hence, $V^{\wedge}(n, k)=0$ for $n=0,1, \ldots ; k=1, \ldots, 2 n+1$. This yields $V=0$ in $\mathcal{A}_{\mathrm{e}}$. By analytical continuation we have $V=0$ in $\mathcal{E}_{\mathrm{e}}$, hence, $v=0$ in $\mathcal{E}_{\mathrm{e}}$. This yields the desired result.

Theorem 3.2 means that the earth's external gravitational field is uniquely recoverable from (negative) 'radial derivatives' corresponding to a fundamental system $\mathcal{X} \subset \mathcal{S}$.

In what follows we mention a uniqueness result related to the oblique derivative problem of potential theory. 
THEOREM 3.3. Let $\mathcal{E}=\mathcal{E}_{\mathrm{i}}$ (i.e. the inner space of the earth) be a regular region. Suppose that $\partial \mathcal{F}$ is the boundary of a regular region $\mathcal{F}=\mathcal{F}_{\mathrm{i}}$ such that $\partial \mathcal{F}$ is a subset of $\mathcal{S}$ (i.e. the satellite orbit). Furthermore, assume that $v$ is of class $\operatorname{pot}^{(0)}\left(\overline{\mathcal{E}_{\mathrm{e}}}\right)$ and $\mathcal{X}$ is a countable dense system of points on $\partial \mathcal{F}$ (i.e. the subset of observational points of the satellite orbit $\mathcal{S}$ ) such that

$$
l(x) \cdot v(x)=0, \quad x \in \mathcal{X},
$$

where $l: \partial \mathcal{F} \rightarrow \mathbb{R}^{3}$ is a unit vector field of class $C^{(0, \lambda)}(\partial \mathcal{F})$ forming with the inner (unit) normal $n$ on $\partial \mathcal{F}$ an angle satisfying $\inf _{x \in \partial \mathcal{F}}(l(x) \cdot n(x))>0$. Then $v=0$ in $\overline{\mathcal{E}_{\mathrm{e}}}$.

Proof. Let $x$ be a point of $\partial \mathcal{F}$ such that $l(x) \cdot v(x) \neq 0$. Because of the continuity of $l \cdot v$ on $\partial F$ there exists a neighbourhood $\mathcal{U}(x)$ such that $l(x) \cdot v(x) \neq 0$ for all $x \in \mathcal{U}(x)$. This, however, contradicts our assumption imposed on the point system $\mathcal{X}$.

The point of departure now is $v=\nabla V$ in $\mathcal{E}_{\mathrm{e}}$. As is well-known in potential theory (see e.g. $[1,11,16]$ ), the oblique derivative problem $V \in \operatorname{Pot}^{(1, \lambda)}\left(\overline{\mathcal{F}_{\mathrm{e}}}\right)$, $(l \cdot \nabla) V=\frac{\partial V}{\partial l}=0$ on $\partial \mathcal{F}, \inf _{x \in \partial \mathcal{F}}(l(x) \cdot n(x))>0$ only has the trivial solution $V=0$ in $\overline{\mathcal{F}_{\mathrm{e}}}$. We therefore get the wanted result in $\overline{\mathcal{E}_{\mathrm{e}}}$ by analytical continuation.

In conclusion, the geopotential field is uniquely determined by a certain set of non-tangential oblique derivatives on the satellite orbit. Moreover, seen from mathematical point of view, lo-lo SST corresponding to an infinite system $\mathcal{X} \subset \mathcal{S}$ delivers redundant information.

From potential theory it is clear that analogous uniqueness theorems (as mentioned before) cannot be deduced for the 'actual' hi-lo SST problem of finding the external gravitational field of the earth from a finite subsystem $\mathcal{X}$ on the satellite orbit $\mathcal{S}$. In what follows, however, we would like to show that, given the SST data for a finite subset $\mathcal{X} \subset \mathcal{S}$, we are able to find, for every value $\varepsilon>0$, an approximation $u$ of the external gravitational field $v$ of the earth in $\varepsilon$-accuracy so that $u$ additionally is consistent to the SST data on the finite subsystem $\mathcal{X}$.

\section{Scalar Approximation}

Let $\partial \mathcal{B}_{\mathrm{e}}$ be a sphere inside (the earth) $\mathcal{E}=\mathcal{E}_{\mathrm{i}}$ of radius $\beta$ centered at the origin 0 (cf. Figure 2) with

$$
\beta<\inf _{x \in \partial \mathcal{E}_{\mathrm{e}}}|x|
$$




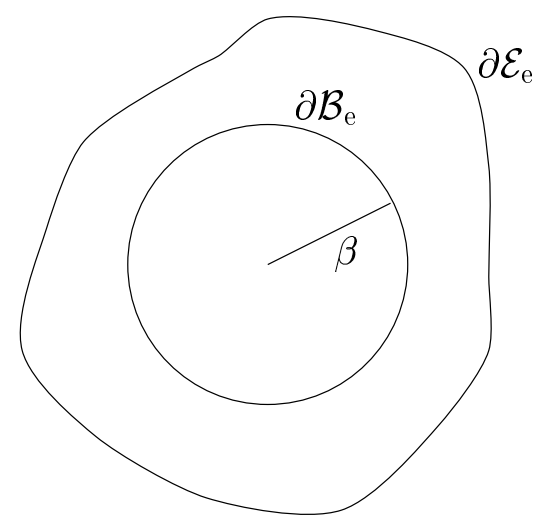

Figure 2: Illustration of the sets $\partial \mathcal{B}_{\mathrm{e}}$ and $\partial \mathcal{E}_{\mathrm{e}}$

We consider simultaneously the outer space $\mathcal{B}_{\mathrm{e}}$ of the sphere $\partial \mathcal{B}_{\mathrm{e}}$ and the outer space $\mathcal{E}_{\text {e }}$ Of course, $\overline{\mathcal{E}_{\mathrm{e}}} \subset \mathcal{B}_{\mathrm{e}}$.

A system $\left(\Phi_{n}\right), \Phi_{n} \in L^{2}\left(\partial \mathcal{B}_{\mathrm{e}}\right), n=0,1, \ldots$, is called complete in the Hilbert space $L^{2}\left(\partial \mathcal{B}_{\mathrm{e}}\right)$, if it satisfies the following property:

(P1) For every $\Phi \in L^{2}\left(\partial \mathcal{B}_{\mathrm{e}}\right)$, the condition

$$
<\Phi, \Phi_{n}>_{\partial \mathcal{B}_{\mathrm{e}}}=\int_{\partial \mathcal{B}_{\mathrm{e}}} \Phi(x) \Phi_{n}(x) d S=0
$$

for all $n=0,1, \ldots$ implies $\Phi=0$ (in the sense of $L^{2}\left(\partial \mathcal{B}_{\mathrm{e}}\right)$ ).

In scalar potential theory a large number of systems $\left(\tilde{\Phi}_{n}\right)_{n=0,1, \ldots}$ is known satisfying $\tilde{\Phi}_{n} \in \operatorname{Pot}^{(0)}\left(\overline{\mathcal{B}_{\mathrm{e}}}\right), \tilde{\Phi}_{n} \mid \partial \mathcal{B}_{\mathrm{e}}=\Phi_{n}, n=0,1, \ldots$, and $\left(\Phi_{n}\right)_{n=0,1, \ldots}$ is complete in $L^{2}\left(\partial \mathcal{B}_{\mathrm{e}}\right)$ (see, for example, $[5,6,10,11]$ ).

The most important system in the geosciences is the system of solid spherical harmonics (i.e. multi-poles).

EXAMPLE 4.1. Let $\left(H_{n, k}(\beta ; \cdot)\right) \underset{\substack{n=0,1, \ldots \\ k=1, \ldots, 2 n+1}}{\substack{n=1 \\ \text { be }}}$ be the system of solid spherical harmonics given by

$$
H_{n, k}(\beta ; x)=\frac{1}{\beta}\left(\frac{\beta}{|x|}\right)^{n+1} Y_{n, k}\left(\frac{x}{|x|}\right), \quad x \in \overline{\mathcal{B}_{\mathrm{e}}} .
$$


Then

$$
\left(\left.H_{n, k}(\beta ; x)\right|_{x \in \partial \mathcal{B}_{\mathrm{e}}}\right)_{n=0,1, \ldots}
$$

is a linearly independent complete system in $L^{2}\left(\partial \mathcal{B}_{\mathrm{e}}\right)$.

In order to illustrate the role of single poles we use the concept of fundamental systems.

EXAmPle 4.2. Suppose that $\mathcal{Y}=\left(y_{n}\right)_{n=0,1, \ldots}$ is a fundamental system in $\mathcal{B}_{\mathrm{i}}$. Denote by

$$
M\left(x, y_{n}\right)=\frac{1}{\left|x-y_{n}\right|}, \quad x \in \overline{\mathcal{B}_{\mathrm{e}}},
$$

the single-poles (mass-points) at $y_{n} \in \mathcal{Y}, n=0,1, \ldots$

Then

$$
\left(\left.M\left(x, y_{n}\right)\right|_{x \in \mathcal{B} \mathcal{B}_{\mathrm{e}}}\right)_{n=0,1, \ldots}
$$

is a linearly independent complete system in $L^{2}\left(\partial \mathcal{B}_{\mathrm{e}}\right)$.

It should be mentioned that the completeness of solid spherical harmonics in $L^{2}\left(\partial \mathcal{B}_{\mathrm{e}}\right)$ is a well-known fact in potential theory (see, for example, [8, 14, 15, 21]). For mass-point systems the property (P1) has been proved already in [5] (in fact, the property $(\mathrm{P} 1)$ can be verified even for arbitrary fundamental systems $\left(y_{n}\right)$ in $\mathcal{E}_{\mathrm{i}}$ and (general) boundaries $\partial \mathcal{B}_{\mathrm{e}}$ of regular regions).

Some examples of fundamental systems in $\mathcal{B}_{\mathrm{i}}$ should be listed below:

(i) If $\mathcal{Y}$ is a countable dense set of points on the boundary $\partial \mathcal{D}_{\mathrm{i}}$ of a regular region $\mathcal{D}_{\mathrm{i}} \subset \mathcal{B}_{\mathrm{i}}$, then $\mathcal{Y}$ is a fundamental system in $\mathcal{B}_{\mathrm{i}}$.

(ii) If $\mathcal{Y}$ is a countable dense set of points in a regular region $\mathcal{D}_{\mathrm{i}} \subset \mathcal{B}_{\mathrm{i}}$, then $\mathcal{Y}$ is a fundamental system in $\mathcal{B}_{\mathrm{i}}$.

(iii) Let $x_{0}$ be a point of $\mathcal{B}_{\mathrm{i}}$ and $\mathcal{Y}=\left(y_{n}\right)_{n=0,1, \ldots} \subset \mathcal{B}_{\mathrm{i}}$ be an infinite sequence of points (with $y_{n} \neq y_{k}$ for all $n \neq k ; n, k=0,1, \ldots$ ) converging to $x_{0}$, then $\mathcal{Y}$ is a fundamental system in $\mathcal{B}_{\mathrm{i}}$.

Remark. Consider the fundamental system $\mathcal{Y}=\left(y_{n}\right)_{n=0,1, \ldots}$ in $\mathcal{B}_{\text {i }}$ generated by $\overline{\mathcal{Y}}=\left(\bar{y}_{n}\right)_{n=0,1, \ldots}$ as follows:

$(\alpha)\left(\bar{y}_{n}\right)$ is a countable dense system on the (real earth's) surface $\partial \mathcal{E} \subset \mathcal{B}_{\mathrm{e}}$,

$(\beta)\left(y_{n}\right)_{n=0,1, \ldots}$ is obtained by letting $y_{n}=\frac{\beta^{2}}{\left|\overline{y_{n}}\right|^{2}} \bar{y}_{n}$. 
This set seems to be a suitable point system for practical purposes (cf. the numerical experiments in $[6])$.

Further complete systems can be obtained by using $\left(K\left(x, y_{n}\right)\right)_{n=0,1, \ldots}$ with

$$
K(x, y)=\frac{1}{|x|} \sum_{k=0}^{\infty} \frac{2 k+1}{4 \pi \beta^{2}} \sigma_{k}\left(\frac{|y|}{|x|}\right)^{k} P_{k}\left(\frac{x}{|x|} \cdot \frac{y}{|y|}\right), x \in \overline{\mathcal{B}_{\mathrm{e}}}, y \in \mathcal{Y} \subset \mathcal{B}_{\mathrm{i}}
$$

instead of the system $\left(M\left(x, y_{n}\right)\right)_{n=0,1, \ldots}$ with

$$
M(x, y)=\frac{1}{|x|} \sum_{k=0}^{\infty}\left(\frac{|y|}{|x|}\right)^{k} P_{k}\left(\frac{x}{|x|} \cdot \frac{y}{|y|}\right), x \in \overline{\mathcal{B}_{\mathrm{e}}}, y \in \mathcal{Y} \subset \mathcal{B}_{\mathrm{i}},
$$

provided that $\mathcal{Y}$ is a fundamental system in $\mathcal{B}_{\mathrm{i}}$ with $\kappa=\sup _{y \in \mathcal{Y}}|y|<\beta$, and the coefficients $\sigma_{k}, \sigma_{k} \neq 0$ for $k=0,1, \ldots$, have to be chosen in such a way that

$$
\sum_{k=0}^{\infty}(2 k+1)\left|\sigma_{k}\right|\left(\frac{\kappa}{\beta}\right)^{k}<\infty
$$

EXAMPLE 4.3. Suppose that $\mathcal{Y}=\left(y_{n}\right)_{n=0,1, \ldots}$ is a fundamental system in $\mathcal{B}_{\mathrm{i}}$ with $\kappa=\sup _{y \in \mathcal{Y}}|y|<\beta$. Let $K\left(x, y_{n}\right)$ be given by (6) (with coefficients $\sigma_{k}$, $\sigma_{k} \neq 0$ for $k=0,1, \ldots$, satisfying the condition (8). Then

$$
\left(\left.K\left(x, y_{n}\right)\right|_{x \in \partial \mathcal{B}_{\mathrm{e}}}\right)_{n=0,1, \ldots}
$$

is a linearly independent complete system in $L^{2}\left(\partial \mathcal{B}_{\mathrm{e}}\right)$.

The proof of the property (P1) for the system $\left(K\left(x, y_{n}\right)\right)_{n=0,1, \ldots}$ immediately follows from the completeness of the spherical harmonics.

Remark. Of numerical significance are series expansions (6) with explicit (i.e. elementary) representation (as, for example, in the case of (7)).

EXAmPle 4.4. Let $y_{0}$ be a fixed point in $\mathcal{B}_{\mathrm{i}}$. Denote by $P_{n}^{y_{0}}(x)$ the expression given by

$$
\begin{gathered}
\left.\left(\frac{\partial}{\partial y_{0}}\right)^{\alpha} K\left(x, y_{0}\right)\right|_{[\alpha]=n}, n=0,1, \ldots \\
\left(\alpha: \text { multiindex, }[\alpha]=\alpha_{1}+\alpha_{2}+\alpha_{3},\left(\frac{\partial}{\partial y_{0}}\right)^{\alpha}=\left.\frac{\partial^{[a]}}{\partial y_{1}^{\alpha_{1}} \partial y_{2}^{\alpha_{2}} \partial y_{3}^{\alpha_{3}}}\right|_{y_{0}}\right)
\end{gathered}
$$


Then

$$
\left(\left.\left.\left(\frac{\partial}{\partial y_{0}}\right)^{\alpha} K\left(x, y_{0}\right)\right|_{[\alpha]=n}\right|_{x \in \partial \mathcal{B}_{\mathrm{e}}}\right)_{n=0,1, \ldots}
$$

is a linearly independent complete system in $L^{2}\left(\partial \mathcal{B}_{\mathrm{e}}\right)$.

The proof follows from Maxwell's representation theorem. (cf. e.g. [8])

Applying the Kelvin transform with respect to the sphere $\partial \mathcal{B}_{\mathrm{e}}$ with radius $\beta$ around the origin (cf. e.g. [14]) Example 4.3 leads us to systems

$$
\left(\left.\bar{K}\left(x, \overline{y_{n}}\right)\right|_{x \in \overline{\mathcal{B}_{\mathrm{e}}}}\right)_{n=0,1, \ldots}
$$

with

$$
\bar{K}(x, \bar{y})=\sum_{k=0}^{\infty} \frac{2 k+1}{4 \pi \beta^{2}} \sigma_{k}\left(\frac{\beta^{2}}{|x||\bar{y}|}\right)^{k+1} P_{k}\left(\frac{x}{|x|} \cdot \frac{\bar{y}}{|\bar{y}|}\right), x \in \overline{\mathcal{B}_{\mathrm{e}}}, \bar{y} \in \overline{\mathcal{Y}} \subset \overline{\mathcal{B}_{\mathrm{e}}}
$$

where $\overline{\mathcal{Y}}=\left(\bar{y}_{n}\right)_{n=0,1, \ldots}$ is the point system generated by $\mathcal{Y}$ by letting $\bar{y}_{n}=$ $\frac{\beta^{2}}{\left|y_{n}\right|^{2}} y_{n}, n=0,1, \ldots$ (thereby assuming $\left.0 \notin \mathcal{Y}\right)$.

Remark. Note that our assumptions imply the estimate

$$
\sum_{k=0}^{\infty}(2 k+1)\left|\sigma_{k}\right|\left(\frac{\beta}{\bar{\kappa}}\right)^{k}<\infty
$$

where $\bar{\kappa}$ is given by

$$
\bar{\kappa}=\inf _{\bar{y} \in \overline{\mathcal{Y}}}|\bar{y}|>\beta
$$

EXAMPLE 4.5. Suppose that $\overline{\mathcal{Y}}=\left(\bar{y}_{n}\right)_{n=0,1, \ldots}$ is given as described above. Let $\bar{K}\left(x, \bar{y}_{n}\right)$ be given as above (with coefficients $\sigma_{k}, \sigma_{k} \neq 0$ for $k=0,1, \ldots$, satisfying (8)). Then

$$
\left(\left.\bar{K}\left(x, \bar{y}_{n}\right)\right|_{x \in \partial \mathcal{B}_{\mathrm{e}}}\right)_{n=0,1, \ldots}
$$

is a linearly independent complete system in $L^{2}\left(\partial \mathcal{B}_{\mathrm{e}}\right)$. 
Typical examples of this type are known from harmonic spline theory $[6,7]$ and geodetic implementations (see [22] and the references therein). We only mention:

(i) Abel-Poisson kernel:

$$
\sigma_{k}=1, \quad k=0,1, \ldots
$$

The kernel reads as follows:

$$
\bar{K}(x, \bar{y})=\frac{1}{4 \pi} \frac{|x|^{2}|\bar{y}|^{2}-\beta^{2}}{(L(x, \bar{y}))^{3 / 2}}, \quad x \in \overline{\mathcal{B}_{\mathrm{e}}}, \bar{y} \in \overline{\mathcal{Y}} \subset \overline{\mathcal{B}_{\mathrm{e}}},
$$

where we have introduced the abbreviation

$$
L(x, \bar{y})=|x|^{2}|\bar{y}|^{2}-2 \beta^{2} x \cdot \bar{y}+\beta^{4} .
$$

(ii) Singularity kernel:

$$
\sigma_{n}=\frac{2}{2 n+1}, \quad n=0,1, \ldots
$$

The kernel is given by

$$
\bar{K}(x, \bar{y})=\frac{1}{2 \pi} \frac{1}{(L(x, \bar{y}))^{1 / 2}}, \quad x \in \overline{\mathcal{B}_{\mathrm{e}}}, \bar{y} \in \overline{\mathcal{Y}} \subset \overline{\mathcal{B}_{\mathrm{e}}} .
$$

(iii) Logarithmic kernel:

$$
\sigma_{n}=\frac{1}{(n+1)(2 n+1)}, \quad n=0,1, \ldots .
$$

Now we have

$$
\bar{K}(x, \bar{y})=\frac{1}{4 \pi \beta^{2}} \ln \left(\frac{\beta^{2}-x \cdot \bar{y}+(L(x, \bar{y}))^{1 / 2}}{|x||\bar{y}|+x \cdot \bar{y}}\right), x \in \overline{B_{\mathrm{e}}}, \bar{y} \in \overline{\mathcal{Y}} \subset \overline{\mathcal{B}_{\mathrm{e}}} .
$$

Remark. Choosing (instead of (8) and (9)) $\sigma_{k}, \sigma_{k} \neq 0$ for $k=0,1, \ldots$, in such a way that

$$
\sum_{k=0}^{\infty}(2 k+1)\left|\sigma_{k}\right|<\infty
$$


i.e. $\left(\left|\sigma_{k}\right|^{-1 / 2}\right)_{k=0,1, \ldots}$ is assumed to be summable (in the sense of [8]), $\kappa$ and $\bar{\kappa}$ are allowed to satisfy $\kappa \leq \beta$ and $\bar{\kappa} \geq \beta$, respectively.

An equivalent statement to the completeness of a system $\left(\Phi_{n}\right)_{n=0,1, \ldots}$ in the space $L^{2}\left(\partial \mathcal{B}_{\mathrm{e}}\right)$ is the closure (see e.g. [2] for the proof of the equivalence).

(P2) For a given function $\Phi \in L^{2}\left(\partial \mathcal{B}_{\mathrm{e}}\right)$ and arbitrary $\varepsilon>0$, there exist an integer $N(=N(\varepsilon))$ and constants $a_{0}, \ldots, a_{N}$ such that

$$
\left(\int_{\partial \mathcal{B}_{\mathrm{e}}}\left|\Phi(x)-\sum_{n=0}^{N} a_{n} \Phi_{n}(x)\right|^{2} d S\right)^{1 / 2} \leq \varepsilon
$$

The property (P2) particularly means that any $\Phi \in C^{(0)}\left(\partial \mathcal{B}_{\mathrm{e}}\right)$ can be approximated by a member of the span of $\left(\Phi_{n}\right)_{n=0,1, \ldots}$ in the sense of the $L^{2}$-metric on $\partial \mathcal{B}_{\mathrm{e}}$.

The step from approximation on the sphere $\partial \mathcal{B}_{\mathrm{e}}$ to approximation in the outer space $\mathcal{B}_{\mathrm{e}}$ can be performed by the following theorem:

THEOREM 4.6. Let $\mathcal{K}$ be a (not necessarily compact) subset of the space $\overline{\mathcal{B}_{\mathrm{e}}}$ with $\operatorname{dist}\left(\mathcal{K}, \partial \mathcal{B}_{\mathrm{e}}\right) \geq \tau>0$. Suppose that $\tilde{\Phi}, \tilde{\Psi}$ are functions of class $\operatorname{Pot}^{(0)}\left(\overline{\mathcal{B}_{\mathrm{e}}}\right)$ with $\tilde{\Phi}\left|\partial \mathcal{B}_{\mathrm{e}}=\Phi, \tilde{\Psi}\right| \partial \mathcal{B}_{\mathrm{e}}=\Psi$. Then there exists a positive constant $C=C\left(\mathcal{K}, \partial \mathcal{B}_{\mathrm{e}}\right)$ such that

$$
\sup _{x \in \mathcal{K}}|\tilde{\Phi}(x)-\tilde{\Psi}(x)| \leq C\left(\int_{\partial \mathcal{B}_{\mathrm{e}}}|\Phi(y)-\Psi(y)|^{2} d S\right)^{1 / 2} .
$$

Proof. Theorem 4.6 is easily verified by application of the Poisson integral formula

$$
\tilde{\Phi}(x)-\tilde{\Psi}(x)=\int_{\partial \mathcal{B}_{\mathrm{e}}} P(x, y)(\Phi(y)-\Psi(y)) d S(y),
$$

where $P(x, y)$ denotes the Abel-Poisson kernel (see e.g. [14]). Put

$$
C=C\left(\mathcal{K}, \partial \mathcal{B}_{\mathrm{e}}\right)=\sup _{x \in \mathcal{K}}\left(\int_{\mathcal{B}_{\mathrm{e}}}|P(x, y)|^{2} d S(y)\right)^{1 / 2}
$$

Then, for each $x \in \mathcal{K}$, the Cauchy-Schwarz inequality yields

$$
|\tilde{\Phi}(x)-\tilde{\Psi}(x)|^{2} \leq C^{2} \int_{\partial \mathcal{B}_{\mathrm{e}}}|\Phi(y)-\Psi(y)|^{2} d S(y) .
$$


This is the desired result.

Let $\tilde{\Phi} \in \operatorname{Pot}^{(0)}\left(\overline{\mathcal{B}_{\mathrm{e}}}\right)$ with $\tilde{\Phi} \mid \partial \mathcal{B}_{\mathrm{e}}=\Phi$. If now $\left(\tilde{\Phi}_{n}\right)_{n=0,1, \ldots} \subset \operatorname{Pot}^{(0)}\left(\overline{\mathcal{B}_{\mathrm{e}}}\right)$ is given such that $\tilde{\Phi}_{n} \mid \partial \mathcal{B}_{\mathrm{e}}=\Phi_{n}, n=0,1, \ldots$, forms a complete system in $L^{2}\left(\partial \mathcal{B}_{\mathrm{e}}\right)$, then for every $\varepsilon>0$ there exist an integer $N(=N(\varepsilon))$ and coefficients $a_{0}, \ldots, a_{N}$ such that

$$
\begin{aligned}
& \sup _{x \in \mathcal{K}}\left|\tilde{\Phi}(x)-\sum_{n=0}^{N} a_{n} \tilde{\Phi}_{n}(x)\right| \\
& \leq C\left(\int_{\partial \mathcal{B}_{\mathrm{e}}}\left|\Phi(y)-\sum_{n=0}^{N} a_{n} \Phi_{n}(y)\right|^{2} d S(y)\right)^{1 / 2} \\
& \leq C \varepsilon
\end{aligned}
$$

for each subset $\mathcal{K} \subset \mathcal{B}_{\mathrm{e}}$ with $\operatorname{dist}\left(\mathcal{K}, \partial \mathcal{B}_{\mathrm{e}}\right) \geq \tau>0$. In other words, given $\tilde{\Phi} \in \operatorname{Pot}^{(0)}\left(\overline{\mathcal{B}_{\mathrm{e}}}\right)$ with $\tilde{\Phi} \mid \partial \mathcal{B}_{\mathrm{e}}=\Phi$, the $L^{2}$-approximation of the function $\Phi$ on the surface $\partial \mathcal{B}_{\mathrm{e}}$ implies ordinary pointwise approximation of $\tilde{\Phi}$ by the system $\left(\tilde{\Phi}_{n}\right)_{n=0,1, \ldots}$ on each subset $\mathcal{K}$ of $\mathcal{B}_{\mathrm{e}}$ with positive distance to $\partial \mathcal{B}_{\mathrm{e}}$.

The system $\left(\tilde{\Phi}_{n}\right)$ is a 'basis system' (more precisely: scalar basis system) in the following sense:

(P3) Each $\tilde{\Phi} \in \operatorname{Pot}^{(0)}\left(\overline{\mathcal{B}_{\mathrm{e}}}\right)$ can be approximated, uniformly on subsets of $\mathcal{B}_{\mathrm{e}}$ with positive distance to $\partial \mathcal{B}_{\mathrm{e}}$, by finite linear combinations of $\left(\tilde{\Phi}_{n}\right)_{n=0,1, \ldots} \subset$ $\operatorname{Pot}^{(0)}\left(\overline{\mathcal{B}_{\mathrm{e}}}\right)$, i.e. for every function $\tilde{\Phi} \in \operatorname{Pot}^{(0)}\left(\overline{\mathcal{B}_{\mathrm{e}}}\right)$ there exists a member $U \in$ $\operatorname{span}_{n=0,1, \ldots}\left(\tilde{\Phi}_{n}\right)$ in $\varepsilon$-accuracy (with respect to the $C^{(0)}$-norm) on every set $\mathcal{K}$ with $\operatorname{dist}\left(\mathcal{K}, \partial \mathcal{B}_{\mathrm{e}}\right) \geq \tau>0$.

As particular case we mention

$$
\sup _{x \in \overline{\mathcal{E}_{\mathrm{e}}}}\left|\tilde{\Phi}(x)-\sum_{n=0}^{N} a_{n} \tilde{\Phi}_{n}(x)\right| \leq C \varepsilon .
$$

\section{Vectorial Approximation}

Let $\mathcal{K}$ be a compact subset of $\mathcal{B}_{\mathrm{e}}$. Since $\mathcal{B}_{\mathrm{e}}$ is assumed to be an open set, $\mathcal{K}$ has a positive distance to the boundary $\partial \mathcal{B}_{\mathrm{e}}$. Hence, there exists a regular region $\mathcal{K}^{*}$ with $\mathcal{K} \subset \mathcal{K}_{\mathrm{e}}^{*}$ and $\overline{\mathcal{K}_{\mathrm{e}}^{*}} \subset \mathcal{B}_{\mathrm{e}}$ (cf. Figure 3 ). 


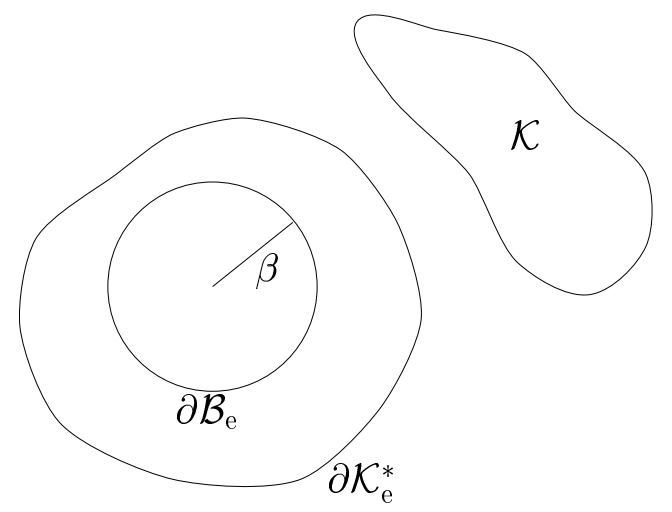

Figure 3: Illustration of the sets $\partial \mathcal{B}_{\mathrm{e}}, \mathcal{K}$ and $\mathcal{K}_{\mathrm{e}}^{*}$

Given $\tilde{\Phi} \in \operatorname{Pot}^{(0)}\left(\overline{\mathcal{B}_{\mathrm{e}}}\right)$, we have

$$
\sup _{x \in \mathcal{K}}|(\nabla \tilde{\Phi})(x)|=\sup _{x \in \mathcal{K}}\left|\nabla_{x} \int_{\partial \mathcal{K}_{\mathrm{e}}^{*}} \tilde{\Phi}(y) \frac{\partial}{\partial n(y)} G^{*}(x, y) d S(y)\right|,
$$

where $G^{*}$ denotes Green's function for the scalar Dirichlet problem (cf. e.g. [14]) in $\mathcal{K}_{\mathrm{e}}^{*}$. Consequently, it follows that

$$
\sup _{x \in \mathcal{K}}|(\nabla \tilde{\Phi})(x)| \leq \sup _{x \in \partial \mathcal{K}_{\mathrm{e}}^{*}}|\tilde{\Phi}(x)| \sup _{x \in \mathcal{K}} \int_{\partial \mathcal{K}_{\mathrm{e}}^{*}}\left|\nabla_{x} \frac{\partial}{\partial n(y)} G^{*}(x, y)\right| d S(y) .
$$

Setting

$$
C^{*}=C^{*}\left(\mathcal{K}, \partial \mathcal{K}_{\mathrm{e}}^{*}\right)=\sup _{x \in \mathcal{K}} \int_{\partial \mathcal{K}_{\mathrm{e}}^{*}}\left|\nabla_{x} \frac{\partial}{\partial n(y)} G^{*}(x, y)\right| d S(y)
$$

we find

$$
\sup _{x \in \mathcal{K}}|(\nabla \tilde{\Phi})(x)| \leq C^{*} \sup _{x \in \partial \mathcal{K}_{\mathrm{e}}^{*}}|\tilde{\Phi}(x)| .
$$

Since $\partial \mathcal{K}_{\mathrm{e}}^{*}$ is a compact set in $\mathcal{B}_{\mathrm{e}}$, we are able to deduce the following statement: THEOREM 5.1. Each scalar basis system $\left(\tilde{\Phi}_{n}\right)_{n=0,1, \ldots}$ i.e. each system $\left(\tilde{\Phi}_{n}\right) \subset$ $\operatorname{Pot}^{(0)}\left(\overline{\mathcal{B}_{\mathrm{e}}}\right)$, where $\left(\tilde{\Phi}_{n} \mid \partial \mathcal{B}_{\mathrm{e}}\right)$ is complete in $L^{2}\left(\partial \mathcal{B}_{\mathrm{e}}\right)$, implies a 'vectorial basis system' in the following sense: For $v \in \operatorname{pot}\left(\mathcal{B}_{\mathrm{e}}\right)$, there exists an approximation 
by a finite linear combination of vector fields $\left(\nabla \tilde{\Phi}_{n}\right)_{n=0,1, \ldots}$, uniformly on compact subsets of $\mathcal{B}_{\mathrm{e}}$.

Proof. Suppose that $v$ is of $\operatorname{class} \operatorname{pot}\left(\mathcal{B}_{\mathrm{e}}\right)$ and $\mathcal{K}$ is an infinite compact subset of $\mathcal{B}_{\mathrm{e}}$. Then there exists a function $V \in \operatorname{Pot}\left(\mathcal{B}_{\mathrm{e}}\right)$ such that $v|\mathcal{K}=(\nabla V)| \mathcal{K}$. Now, for arbitrary $\varepsilon>0$, we have an integer $N(=N(\varepsilon))$ and coefficients $a_{0}, \ldots, a_{N}$ such that

$$
\sup _{x \in \partial \mathcal{K}_{\varepsilon}^{*}}\left|V(x)-\sum_{n=0}^{N} a_{n} \tilde{\Phi}_{n}(x)\right| \leq \varepsilon .
$$

In connection with (15) this gives us

$$
\begin{aligned}
\sup _{x \in \mathcal{K}}\left|v(x)-\sum_{n=0}^{N} a_{n}\left(\nabla \tilde{\Phi}_{n}\right)(x)\right| & \leq C^{*} \sup _{x \in \partial \mathcal{K}_{\varepsilon}^{*}}\left|V(x)-\sum_{n=0}^{N} a_{n} \tilde{\Phi}_{n}(x)\right| \\
& \leq C^{*} \varepsilon .
\end{aligned}
$$

This is the desired result.

Next we discuss some relations between the spaces $\operatorname{pot}\left(\mathcal{B}_{\mathrm{e}}\right) \mid \overline{\mathcal{E}_{\mathrm{e}}}$ and $\operatorname{pot}^{(0, \lambda)}\left(\overline{\mathcal{E}_{\mathrm{e}}}\right)$. Of course, we have

$$
\operatorname{pot}\left(\mathcal{B}_{\mathrm{e}}\right) \mid \overline{\mathcal{E}_{\mathrm{e}}} \subset \operatorname{pot}^{(0, \lambda)}\left(\overline{\mathcal{E}_{\mathrm{e}}}\right)
$$

The inclusion is, in fact, strict: choose $y \in \mathcal{B}_{\mathrm{e}} \backslash \overline{\mathcal{E}_{\mathrm{e}}}$, then the field

$$
x \mapsto \nabla_{x} \frac{1}{|x-y|}, \quad x \neq y,
$$

is an element of class $\operatorname{pot}^{(0, \lambda)}\left(\overline{\mathcal{E}_{\mathrm{e}}}\right)$, but it is obvious that the vector field is not an element of $\operatorname{pot}\left(\mathcal{B}_{\mathrm{e}}\right) \mid \overline{\mathcal{E}_{\mathrm{e}}}$. Hence,

$$
\operatorname{pot}\left(\mathcal{B}_{\mathrm{e}}\right) \mid \overline{\mathcal{E}_{\mathrm{e}}} \neq \operatorname{pot}^{(0, \lambda)}\left(\overline{\mathcal{E}_{\mathrm{e}}}\right) .
$$

However, we are able to prove the following closure theorem:

THEOREM 5.2. The space $\operatorname{pot}\left(\mathcal{B}_{\mathrm{e}}\right) \mid \overline{\mathcal{E}_{\mathrm{e}}}$ is a dense subset of the space $\operatorname{pot}^{(0, \lambda)}\left(\overline{\mathcal{E}_{\mathrm{e}}}\right)$ with respect to \|\|$_{\lambda}\left(\overline{\mathcal{E}_{\mathrm{e}}}\right)$, i.e. for any given value $\varepsilon>0$ and any element $v \in \operatorname{pot}^{(0, \lambda)}\left(\overline{\mathcal{E}_{\mathrm{e}}}\right)$ there exists a field $u \in \operatorname{pot}\left(\mathcal{B}_{\mathrm{e}}\right) \mid \overline{\mathcal{E}_{\mathrm{e}}}$ such that ${ }^{1}$

$$
\|v-u\|_{\lambda}\left(\overline{\mathcal{E}_{\mathrm{e}}}\right) \leq \varepsilon .
$$

\footnotetext{
${ }^{1}$ Note that the norm symbols are used simultaneously for scalar- and vector-valued functions.
} 
Proof. The main tool is the classical Hahn-Banach Theorem. Let $F$ be a linear functional on $\operatorname{pot}^{(0, \lambda)}\left(\overline{\mathcal{E}_{\mathrm{e}}}\right)$, continuous with respect to \|\|$_{\lambda}\left(\overline{\mathcal{E}_{\mathrm{e}}}\right)$. Assume that $F$ is zero on the set $\operatorname{pot}\left(\mathcal{B}_{\mathrm{e}}\right) \mid \overline{\mathcal{E}_{\mathrm{e}}}$. Then we have to prove that $F$ is the zero functional, since this argumentation implies that $\operatorname{pot}\left(\mathcal{B}_{\mathrm{e}}\right) \mid \overline{\mathcal{E}_{\mathrm{e}}}$ is dense in $\operatorname{pot}^{(0, \lambda)}\left(\overline{\mathcal{E}_{\mathrm{e}}}\right)$ with respect to \|\|$_{\lambda}\left(\overline{\mathcal{E}_{\mathrm{e}}}\right)$ due to a theorem in e.g. [13].

Because of the regularity of $\mathcal{E}=\mathcal{E}_{\mathrm{i}}$ we are allowed to construct at each point $x \in \partial \mathcal{E}_{\mathrm{e}}$ a normal $n(x)$ pointing into $\mathcal{E}_{\mathrm{i}}$. From the exterior Neumann problem of potential theory we know that, for a given vector field $f \in C^{(0, \lambda)}\left(\partial \mathcal{E}_{\mathrm{e}}\right)$, there exists a unique vector field $u$ satisfying

$$
u \in \operatorname{pot}^{(0, \lambda)}\left(\overline{\mathcal{E}_{\mathrm{e}}}\right), \quad\left(u \mid \partial \mathcal{E}_{\mathrm{e}}\right) \cdot n=f .
$$

The unique solution $u$ can be represented as gradient field of a potential involving a single layer $G \in C^{(0, \lambda)}\left(\partial \mathcal{E}_{\mathrm{e}}\right)$ (see e.g. $[1,10,11,12,16]$ )

$$
u(x)=\nabla_{x} \int_{\partial \mathcal{E}_{\mathrm{e}}} G(y) \frac{1}{|x-y|} d S(y), \quad x \in \overline{\mathcal{E}_{\mathrm{e}}} .
$$

Moreover, we have the estimate (cf. [20])

$$
\|u\|_{\lambda}\left(\overline{\mathcal{E}_{\mathrm{e}}}\right) \leq C_{\lambda}\left\|\left(u \mid \partial \mathcal{E}_{\mathrm{e}}\right) \cdot n\right\|_{\lambda}\left(\partial \mathcal{E}_{\mathrm{e}}\right) .
$$

For any point $y \in \mathcal{E}_{\mathrm{i}}$, the vector field

$$
x \mapsto a_{y}(x)=\nabla_{y} \frac{1}{|x-y|}, \quad x \neq y,
$$

is of class $\operatorname{pot}^{(0, \lambda)}\left(\overline{\mathcal{E}_{\mathrm{e}}}\right)$. Thus, by setting

$$
A(y)=F\left(a_{y} \mid \overline{\mathcal{E}_{\mathrm{e}}}\right)
$$

we get a function $A$ defined on $\mathcal{E}_{\mathrm{i}}$. The expressions

$$
\left(\varepsilon^{i} \cdot \nabla_{y}\right) a_{y}(x)=\frac{\partial}{\partial y_{i}} a_{y}(x)=\frac{\partial}{\partial y_{i}} \nabla_{y} \frac{1}{|x-y|}, \quad x \in \overline{\mathcal{E}_{\mathrm{e}}}
$$

define vector fields $\frac{\partial}{\partial y_{i}} a_{y}, i=1,2,3$, of class pot ${ }^{(0, \lambda)}\left(\overline{\mathcal{E}_{\mathrm{e}}}\right)$. Furthermore, it is not difficult to see that $A$ is a continuously differentiable function on $\mathcal{E}_{\mathrm{i}}$ satisfying

$$
\frac{\partial}{\partial y_{i}} A(y)=F\left(\frac{\partial}{\partial y_{i}}\left(a_{y} \mid \overline{\mathcal{E}_{\mathrm{e}}}\right)\right)
$$


Hence, obverserving (23) we find

$$
\operatorname{curl} \nabla_{y} A(y)=0, \quad \operatorname{div} \nabla_{y} A(y)=0, \quad y \in \mathcal{E}_{\mathrm{i}} .
$$

This means that $A$ is analytic in $\mathcal{E}_{\mathrm{i}}$. On the other hand, for $y \in \mathcal{B}_{\mathrm{i}}$ it is obvious that $a_{y} \mid \mathcal{B}_{\mathrm{e}} \in \operatorname{pot}\left(\mathcal{B}_{\mathrm{e}}\right)$ and $a_{y}\left|\overline{\mathcal{E}_{\mathrm{e}}} \in \operatorname{pot}\left(\mathcal{B}_{\mathrm{e}}\right)\right| \overline{\mathcal{E}_{\mathrm{e}}}$. Hence, for $y \in \mathcal{B}_{\mathrm{i}}$, $F\left(a_{y} \mid \overline{\mathcal{E}_{\mathrm{e}}}\right)=A(y)=0$. Analytic continuation gives $A(y)=0$ for all points $y \in \mathcal{E}_{\mathrm{i}}$. This yields, in particular,

$$
0=G(x) A(x+\operatorname{sn}(x))=F\left(G(x) a_{x+s n(x)} \mid \overline{\mathcal{E}_{\mathrm{e}}}\right)
$$

for each $x \in \partial \mathcal{E}_{\mathrm{e}}$ and (sufficiently small) $s>0$. The mappings $\tau_{s}: \partial \mathcal{E}_{\mathrm{e}} \rightarrow$ $\operatorname{pot}^{(0, \lambda)}\left(\overline{\mathcal{E}_{\mathrm{e}}}\right)$ given by

$$
\tau_{s}(x)=G(x) a_{x+s n(x)} \mid \overline{\mathcal{E}_{\mathrm{e}}}
$$

will be investigated in parallel to arguments in [10], Appendix 4. First the Hölder continuity of $\tau_{s}$ will be verified. We start with the estimate

$$
\begin{aligned}
\left\|\tau_{s}(x)-\tau_{s}\left(x^{\prime}\right)\right\|_{\lambda}\left(\partial \mathcal{E}_{\mathrm{e}}\right) \leq & \left|G\left(x^{\prime}\right)\right|\left\|a_{x+s n(x)}-a_{x^{\prime}+s n\left(x^{\prime}\right)}\right\|_{\lambda}\left(\partial \mathcal{E}_{\mathrm{e}}\right) \\
& +\left|G(x)-G\left(x^{\prime}\right)\right|\left\|a_{x+s n(x)}\right\|_{\lambda}\left(\partial \mathcal{E}_{\mathrm{e}}\right)
\end{aligned}
$$

for $x, x^{\prime} \in \partial \mathcal{E}_{\mathrm{e}}$.

Explicit calculations in connection with the mean value theorem show us that

$$
\left\|a_{x+s n(x)}\right\|_{\lambda}\left(\partial \mathcal{E}_{\mathrm{e}}\right)=O\left(s^{-3}\right)
$$

as $s \rightarrow 0+$.

The Hölder continuity of $G$ gives

$$
\left|G(x)-G\left(x^{\prime}\right)\right|=O\left(\left|x-x^{\prime}\right|^{\lambda}\right)
$$

as $\left|x-x^{\prime}\right| \rightarrow 0+$.

Moreover,

$$
\begin{aligned}
\left\|a_{x+s n(x)}-a_{x^{\prime}+\operatorname{sn}\left(x^{\prime}\right)}\right\|_{\lambda}\left(\partial \mathcal{E}_{\mathrm{e}}\right) & =O\left(x+\operatorname{sn}(x)-\left(x^{\prime}+s n\left(x^{\prime}\right)\right)\right) \\
& =O\left(\left|x-x^{\prime}\right|+s\left|n(x)-n\left(x^{\prime}\right)\right|\right) \\
& =O\left(\left|x-x^{\prime}\right|^{\lambda}\right) \quad\left(x \rightarrow x^{\prime}\right)
\end{aligned}
$$

where we have used the estimate

$$
\left|n(x)-n\left(x^{\prime}\right)\right| \leq C\left|x-x^{\prime}\right|
$$


(which is certainly true for a $C^{(2, \lambda)}$-smooth surface $\partial \mathcal{E}_{\mathrm{e}}$ ). Summarizing our results $(26),(27)$, and (28) we therefore obtain

$$
\begin{aligned}
\left\|\tau_{s}(x)-\tau_{s}\left(x^{\prime}\right)\right\|_{\lambda}\left(\partial \mathcal{E}_{\mathrm{e}}\right) & =O\left(\|G\|_{0}\left(\partial \mathcal{E}_{\mathrm{e}}\right)\left|x-x^{\prime}\right|^{\lambda}+\frac{1}{s^{3}}\left|x-x^{\prime}\right|^{\lambda}\right) \\
& =O\left(\left|x-x^{\prime}\right|^{\lambda}\right) \quad\left(x \rightarrow x^{\prime}\right)
\end{aligned}
$$

i.e. $x \rightarrow x^{\prime}$ implies $\tau_{s}(x) \rightarrow \tau_{s}\left(x^{\prime}\right)$ with respect to \|\|$_{\lambda}\left(\partial \mathcal{E}_{\mathrm{e}}\right)$, hence, $\tau_{s}$ is continuous with respect to \|\|$_{\lambda}\left(\partial \mathcal{E}_{\mathrm{e}}\right)$, thus integrable over $\partial \mathcal{E}_{\mathrm{e}}$. Now, we have

$$
\begin{aligned}
0 & =\int_{\partial \mathcal{E}_{\mathrm{e}}} G(x) A(x+\operatorname{sn}(x)) d S(x) \\
& =\int_{\partial \mathcal{E}_{\mathrm{e}}} F\left(G(x) a_{x+s n(x)} \mid \overline{\mathcal{E}_{\mathrm{e}}}\right) d S(x) .
\end{aligned}
$$

By virtue of the continuity of $F$ and the integrability of $\tau_{s}$ over $\partial \mathcal{E}_{\mathrm{e}}$ the linear functional and the integral may be interchanged (see also [15]).

$$
0=F\left(\int_{\mathcal{E}_{\mathrm{e}}}\left(G(x) a_{x+s n(x)} \mid \overline{\mathcal{E}_{\mathrm{e}}}\right) d S(x)\right), s>0 .
$$

Using the abbreviation

$$
u_{s}(y)=\left.\int_{\partial \mathcal{E}_{\mathrm{e}}} G(x) \nabla_{z} \frac{1}{|z-y|}\right|_{z=x+\operatorname{sn}(x)} d S(x)
$$

the identity (29) reduces to

$$
0=F\left(u_{s}\right), \quad s>0
$$

The vector field $u_{s}$ is of class pot ${ }^{(0, \lambda)}\left(\overline{\mathcal{E}_{\mathrm{e}}}\right)$ (cf. [20]). In [10], Appendix 5, it has been shown that

$$
\left(u_{s} \mid \partial \mathcal{E}_{\mathrm{e}}\right) \cdot n \rightarrow\left(u \mid \partial \mathcal{E}_{\mathrm{e}}\right) \cdot n, \quad s \rightarrow 0,
$$

with respect to \|\|$_{\lambda}\left(\partial \mathcal{E}_{\mathrm{e}}\right)$. Therefore, by virtue of (19), we have

$$
u_{s} \rightarrow u, \quad s \rightarrow 0
$$


with respect to \|\|$_{\lambda}\left(\overline{\mathcal{E}_{\mathrm{e}}}\right)$. The continuity of $F$ then shows

$$
F(u)=\lim _{\substack{s \rightarrow 0 \\ s>0}} F\left(u_{s}\right)=0,
$$

as required.

Finally, the closure theorem (Theorem 5.2) enables us to derive the following approximation theorem:

THEOREM 5.3. Let $\left(\tilde{\Phi}_{n}\right)_{n=0,1, \ldots}$ be a system of functions $\tilde{\Phi}_{n} \in \operatorname{Pot}^{(0)}\left(\overline{\mathcal{B}_{\mathrm{e}}}\right)$, $n=0,1, \ldots$, such that $\left(\tilde{\Phi}_{n} \mid \partial \mathcal{B}_{\mathrm{e}}\right)_{n=0,1, \ldots}$ is complete in $L^{2}\left(\partial \mathcal{B}_{\mathrm{e}}\right)$. Then, every function $v \in \operatorname{pot}^{(0, \lambda)}\left(\overline{\mathcal{E}_{\mathrm{e}}}\right)$ can be approximated in the metric \|\|$_{\lambda}\left(\overline{\mathcal{E}_{\mathrm{e}}}\right)$ by a finite linear combination of the gradient fields $\left(\nabla \tilde{\Phi}_{n}\right)_{n=0,1, \ldots}$, i.e. for given $\varepsilon>0$ and $v \in \operatorname{pot}^{(0, \lambda)}\left(\overline{\mathcal{E}_{\mathrm{e}}}\right)$, there exist an integer $N(=N(\varepsilon))$ and coefficients $a_{0}, \ldots, a_{N}$ such that

$$
\left\|v-\sum_{n=0}^{N} a_{n} \nabla \tilde{\Phi}_{n}\right\|_{\lambda}\left(\overline{\mathcal{E}_{\mathrm{e}}}\right) \leq \varepsilon .
$$

Proof. In comparison to Theorem 5.2 it remains to prove that any continuous linear functional $F$ on $\operatorname{pot}^{(0, \lambda)}\left(\overline{\mathcal{E}_{\mathrm{e}}}\right)$ satisfying $F\left(\nabla \tilde{\Phi}_{n} \mid \overline{\mathcal{E}_{\mathrm{e}}}\right)=0$ for $n=0,1, \ldots$, is zero on the set $\operatorname{pot}\left(\mathcal{B}_{\mathrm{e}}\right) \mid \overline{\mathcal{E}_{\mathrm{e}}}$.

Let $u$ be a vector field of $\operatorname{class} \operatorname{pot}\left(\mathcal{B}_{\mathrm{e}}\right)$. Then there exists a function $U \in$ $\operatorname{Pot}\left(\mathcal{B}_{\mathrm{e}}\right)$ with $u=\nabla U$. Since $\left(\tilde{\Phi}_{n}\right)_{n=0,1, \ldots}$ is assumed to be a scalar basis system in $\overline{\mathcal{B}_{\mathrm{e}}}$, the function $U$ can be approximated by finite linear combinations $U_{N}$ of $\left(\tilde{\Phi}_{n}\right)$, i.e. $U_{N} \rightarrow U$ on each compact subset $\mathcal{K}$ of $\mathcal{B}_{\mathrm{e}}$. A result given in [15] shows that any partial derivative of $U_{N}$ tends to the corresponding partial derivative of $U$ uniformly on each compact set $\mathcal{K}$ of $\mathcal{B}_{\mathrm{e}}$. We consider, in particular, the second order derivatives and a bounded neighbourhood of $\partial \mathcal{E}_{\mathrm{e}}$. Then, by application of the mean value theorem of multidimensional analysis, $\nabla U_{N} \rightarrow \nabla U$ in the Hölder norm \|\|$_{\lambda}\left(\partial \mathcal{E}_{\mathrm{e}}\right)$. Consequently, by (19), we obtain $\nabla U_{N} \rightarrow \nabla U$ in the sense of \|\|$_{\lambda}\left(\overline{\mathcal{E}_{\mathrm{e}}}\right)$. In accordance with the assumption $F\left(\nabla U_{N} \mid \overline{\mathcal{E}_{\mathrm{e}}}\right)=0$. Hence, the continuity of $F$ gives us

$$
F(u)=F\left(\nabla U \mid \overline{\mathcal{E}_{\mathrm{e}}}\right)=\lim _{N \rightarrow \infty} F\left(\nabla U_{N} \mid \overline{\mathcal{E}_{\mathrm{e}}}\right)=0
$$

as required.

In connection with the norm estimate (4) and Theorem 5.3 we find the following corollary. 
COROLlaRY 5.4. Let $\left(\tilde{\Phi}_{n}\right)_{n=0,1, \ldots}$ be a scalar basis system of functions $\tilde{\Phi}_{n} \in$ $\operatorname{Pot}^{(0)}\left(\overline{\mathcal{B}_{\mathrm{e}}}\right), n=0,1, \ldots$, in the sense of the property (P3). Then, for given $\varepsilon>0$ and $v \in \operatorname{pot}^{(0)}\left(\overline{\mathcal{E}_{\mathrm{e}}}\right)$, there exist an integer $N(=N(\varepsilon))$ and coefficients $a_{0}, \ldots, a_{N}$ such that

$$
\left\|v-\sum_{n=0}^{N} a_{n} \nabla \tilde{\Phi}_{n}\right\|_{0}\left(\overline{\mathcal{E}_{\mathrm{e}}}\right) \leq \varepsilon .
$$

Hence, the external gravitational field $v$ of the earth admits a uniform approximation by gradient fields of scalar basis systems of class $\operatorname{Pot}^{(0)}\left(\overline{\mathcal{B}_{\mathrm{e}}}\right)$ on and outside the earth's surface.

From an extended version of the Helly Theorem (see [23]) we are able to derive the following corollaries, which play an important role in hi-lo SST of determining the earth's gravitational field from a finite set of GPS-SST data.

Corollary 5.5. Let the assumptions of Corollary 5.4 be fulfilled. Let $\mathcal{X}$ be a finite subset of $\mathcal{S} \subset \mathcal{E}_{\mathrm{e}}$ satisfying (1). Then, for given $\varepsilon>0$ and $v \in$ $\operatorname{pot}^{(0, \lambda)}\left(\overline{\mathcal{E}_{\mathrm{e}}}\right)$, there exist an integer $N(=N(\varepsilon))$ and coefficients $a_{0}, \ldots, a_{N}$ such that

$$
\left\|v-\sum_{n=0}^{N} a_{n} \nabla \tilde{\Phi}_{n}\right\|_{\lambda}\left(\overline{\mathcal{E}_{\mathrm{e}}}\right) \leq \varepsilon
$$

and

$$
v(x)=\sum_{n=0}^{N} a_{n}\left(\nabla \tilde{\Phi}_{n}\right)(x), \quad x \in \mathcal{X} .
$$

COROLlary 5.6. Let the assumptions of Corollary 5.4 be fulfilled. Let $l$ be a vector field given on a finite subset $\mathcal{X}$ of $\mathcal{S} \subset \mathcal{E}_{\mathrm{e}}$ satisfying (1). Then, for given $\varepsilon>0$ and $v \in \operatorname{pot}^{(0, \lambda)}\left(\overline{\mathcal{E}_{\mathrm{e}}}\right)$, there exist an integer $N(=N(\varepsilon))$ and coefficients $a_{0}, \ldots, a_{N}$ such that

$$
\left\|v-\sum_{n=0}^{N} a_{n} \nabla \tilde{\Phi}_{n}\right\|_{\lambda}\left(\overline{\mathcal{E}_{\mathrm{e}}}\right) \leq \varepsilon
$$

and

$$
l(x) \cdot v(x)=\sum_{n=0}^{N} a_{n} l(x) \cdot\left(\nabla \tilde{\Phi}_{n}\right)(x), \quad x \in \mathcal{X} .
$$

In other words, the geopotential field admits an approximation (in $\varepsilon$-accuracy with respect to the \|\|$_{\lambda}\left(\overline{\mathcal{E}_{\mathrm{e}}}\right)-$ norm) consistent to a finite number of (oblique) derivatives.

Furthermore, lo-lo SST can be based successfully on the following result: 
Corollary 5.7. Let the assumptions of Corollary 5.4 be fulfilled. Let $l$ be a vector field given on a finite subset $\mathcal{X}$ of $\mathcal{S}$ satisfying (1). Then, for given $\varepsilon>0$ and $v \in \operatorname{pot}^{(0, \lambda)}\left(\overline{\mathcal{E}_{\mathrm{e}}}\right)$, there exist an integer $N(=N(\varepsilon))$ and coefficients $a_{0}, \ldots, a_{N}$ such that

$$
\left\|v-\sum_{n=0}^{N} a_{n} \nabla \tilde{\Phi}_{n}\right\|_{\lambda}\left(\overline{\mathcal{E}_{\mathrm{e}}}\right) \leq \varepsilon
$$

and

$$
l(x) \cdot(v(x)-v(x+h(x)))=\sum_{n=0}^{N} a_{n} l(x) \cdot\left(\left(\nabla \tilde{\Phi}_{n}\right)(x)-\left(\nabla \tilde{\Phi}_{n}\right)(x+h(x))\right),
$$

$x \in \mathcal{X}$. In particular,

$h(x) \cdot(v(x)-v(x+h(x)))=\sum_{n=0}^{N} a_{n} h(x) \cdot\left(\left(\nabla \tilde{\Phi}_{n}\right)(x)-\left(\nabla \tilde{\Phi}_{n}\right)(x+h(x))\right)$,

$x \in \mathcal{X}$

Clearly, the Hölder topology in Corollary 5.5, 5.6, and 5.7 can be substituted by the uniform topology on $\overline{\mathcal{E}_{\mathrm{e}}}$ (as indicated in Corollary 5.4 ).

\section{Acknowledgements.}

The support by German Research Foundation (Deutsche Forschungsgemeinschaft, Bonn, Contract No. FR 761/5-1) and Graduiertenkolleg Technomathematik (Universität Kaiserslautern) is greatfully acknowledged.

\section{References}

[1] Bitzadse, A.V. (1968) Boundary Value Problems for Second Order Elliptic Equations. North-Holland Publishing Company, Amsterdam.

[2] Davis, P.J. (1963) Interpolation and Approximation. Blaisdell Publishing Company, New York, Toronto, London.

[3] ESA (1996) The Nine Candidate Earth Explorer Missions. Publications Division ESTEC, Nordwijk, SP-1196 (1).

[4] ESA (1998) European Views on Dedicated Gravity Field Missions: GRACE and GOCE. ESD-MAG-REP-CON-001. 
[5] Freeden, W. (1980) On the Approximation of External Gravitational Potential with Closed Systems of (Trial) Functions. Bull. Geod., 54, 1-20.

[6] Freeden, W. (1987) A Spline Interpolation Method for Solving Boundary Value Problems of Potential Theory from Discretely Given Data. Numer. Meth. Partial Diff. Equations, 3, 375-398.

[7] Freeden, W. (1999): Multiscale Modelling of Spaceborne Geodata, B.G. Teubner, Stuttgart, Leipzig.

[8] Freeden, W., Gervens, T., Schreiner, M. (1998) Constructive Approximation on the Sphere (With Applications to Geomathematics). Oxford Science Publications, Clarendon.

[9] Freeden, W., Glockner, O., Thalhammer, M. (1999) Multiscale Gravitational Field Recovery from GPS-Satellite-to-Satellite Tracking. Studia Geoph. et Geod., 43, 229-264.

[10] Freeden, W., Kersten, H. (1980) The Geodetic Boundary Value Problem Using the Known Surface of the Earth. Veröff. Geod. Inst. RWTH Aachen, Heft 29.

[11] Freeden, W., Kersten, H. (1981) A Constructive Approximation Theorem for the Oblique Derivative Problem in Potential Theory. Math. Meth. Appl. Sci., 3, 104-114.

[12] Giraud, G. (1934) Equations a integrales principales, Etude suivie d'une application. Ann. Sci. Ec. Norm. Sup., 51, 251-372.

[13] Kantorowitsch, L.W., Akilow, G. (1964) Funktionalanalysis in normierten Räumen. Akademie-Verlag, Berlin.

[14] Kellogg, O.D. (1929) Foundations of Potential Theory. Frederick Ungar Publishing Company, New York.

[15] Martensen, E. (1968) Potentialtheorie, Teubner Verlag, Stuttgart.

[16] Miranda, C. (1970) Partial Differential Equations of Elliptic Type. Springer, New York - Heidelberg - Berlin.

[17] Reigber, C. (1980) Gravity Field Recovery from Satellite Tracking Data. In Sanso, Rummel (eds.): Theory of Satellite Geodesy and Gravity Field Determination, Lecture Notes in Earth Sciences, 25, Springer, Berlin. 
[18] Reigber, C., Bock, R., Förste, C., Grunwaldt, L., Jakowski, N., Lühr, H., Schwintzer, P., Tilgner, C. (1996) CHAMP-Phase B, Executive Summary. Scientific Technical Report STR96/13 GFZ Potsdam.

[19] Rummel, R. (1979) Determination of the Short-wavelength Components of the Gravity Field from Satellite-to-Satellite Tracking or Satellite Gradiometry. Manuscr. Geod., 4, 107-148.

[20] Schauder, J. (1931) Potentialtheoretische Untersuchungen, Math. Z., 33, 602-640, Math. Z., 35, 536-538.

[21] Walsh, J.L. (1929) The Approximation of Harmonic Functions by Harmonic Polynomials and by Harmonic Rational Functions. Am. Math. Soc., 35, 499-544.

[22] Tscherning, C.C., Forsberg, R., Vermeer, M. (1990) Methods for Regional Gravity Field Modelling from SST and SGG Data. Reports of the Finnish Geodetic Institute 90:2.

[23] Yamabe, H. (1950) On an Extension of the Helly's Theorem. Osaka Mathematical Journal, 2, No. 1, 15-22. 